\title{
Efecto del Maltrato Físico en la Calidad de las Representaciones de Apego Infantil en Chile: Resultados Preliminares
}

\author{
The Effect of Physical Abuse on The Quality of Infant Attachment \\ Representations in Chile: Preliminary Results
}

\author{
Andrés Fresno* \\ $\&$ \\ Rosario Spencer \\ Universidad de Talca, Chile
}

(Rec: 17 de Junio de 2011 / Acep: 4 de Octubre de 2011)

\begin{abstract}
Resumen
El objetivo de este artículo es presentar los resultados preliminares de un estudio que analiza el efecto del maltrato físico sobre la calidad de las representaciones de apego infantil. Para evaluar las representaciones del apego se utilizó la tarea de completación de historias de apego con el sistema de codificación A.S.C.T. Q-sort. El maltrato físico fue categorizado con el Sistema de Codificación de Maltrato Modificado. Los resultados indican que el maltrato físico tiene un efecto negativo en la calidad de las representaciones del apego. Los niños víctimas de maltrato físico presentan una mayor preferencia por la utilización de estrategias de hiperactivación y desorganización del apego que los niños no maltratados. Los resultados son discutidos en relación a la teoría del apego y a la evidencia empírica sobre esta temática.

Palabras clave: Maltrato físico, representaciones de apego, apego, infancia.
\end{abstract}

\begin{abstract}
The aim of this paper is to present preliminary results of a study analyzing the effects of physical abuse on the quality of attachment representations in children. In order to assess the attachment representation the Attachment Story Completion Task was used with de A.S.C.T. Q-sort coding system. The physical abuse was coded using the Modified Maltreatment Classification System. The results show that physical abuse has a negative effect on the quality of attachment representation. The children who were victims of physical abuse have a higher preference for the use of hyperactivation and disorganization attachment strategies than non abused children. The results are discussed in relation to attachment theory and empirical evidence on this issue.

Key words: Physical abuse, attachment representation, attachment, childhood.
\end{abstract}

* Correspondencia: Andrés Fresno Rodríguez, Facultad de Psicología, Universidad de Talca, 2 norte 685, Talca, Chile. E-mail: afresno@utalca.cl Esta investigación fue financiada por el proyecto FONDECYT N ${ }^{\circ} 11080107$ y contó con el patrocinio de la Universidad de Talca, SENAME y la colaboración de la Fundación CRATE. 


\section{Introducción}

La literatura afirma que el maltrato afecta negativamente el desarrollo de los niños (Cicchetti \& Toth, 2005). Desde la teoría del apego (Bowlby, 1980) los estudios van en la misma dirección, indicando que el maltrato afecta la calidad del apego infantil y su consecuente desarrollo (Baer \& Daly Martinez, 2006; Hodges \& Steele, 2000). No obstante, las investigaciones que han abordado esta temática han estudiado el apego desde una perspectiva comportamental, existiendo escasa literatura respecto al impacto del maltrato sobre las representaciones del apego (Baer \& Daly Martínez, 2006; Toth, Cicchetti, Macfie, Maughan \& Vanmeenen, 2000). Este artículo tiene por objetivo presentar los resultados preliminares de un estudio acerca de la influencia del maltrato en la calidad de las representaciones de apego infantil, particularmente aquellos referidos al efecto específico del maltrato físico.

En Chile el 75,3\% de los niños sufre algún tipo de maltrato por parte de sus padres; de este total, el 53,8\% corresponde a violencia física (UNICEF, 2008). Si bien existen diversas definiciones de maltrato físico, en general existe concordancia en definirlo como aquellas acciones realizadas por el cuidador o adulto responsable que ocasionan lesiones físicas al niño, exceptuando aquellos casos en que las lesiones fueron accidentales (Barnett, Manly, \& Cicchetti, 1993, citados en Cicchetti \& Toth, 1993; UNICEF, 2008).

Desde una perspectiva ecológica transaccional (Cicchetti \& Toth, 2005), la ocurrencia del maltrato puede deberse a la interacción entre factores de riesgo y protectores en distintos niveles ecológicos. En el nivel más alejado de la interacción directa con el niño se pueden encontrar la aceptación cultural y creencias que validan la utilización de la violencia, el castigo físico y la violencia intrafamiliar. A nivel de la comunidad, se pueden dar altos niveles de violencia y delincuencia. En al ámbito familiar, los factores de riesgo estarían dados por las características de sus miembros que favorezcan el maltrato. Estos factores, más el incremento de estresores tales como bajo nivel de ingreso, cesantía, peligrosidad del barrio, condiciones habitacionales inadecuadas, entre otros, afectan negativamente las prácticas parentales, aumentando el riego de maltrato hacia los niños (Elder, Van Nguyen \& Caspi, 1985; McLoyd, 1990).

Respecto al maltrato físico, la literatura científica es clara en señalar que tiene un efecto negativo sobre el desarrollo de los niños a nivel físico, cognitivo, social y emocional (Cicchetti \& Toth, 2005; Howe, 2005). Desde los estudios basados en la teoría del apego (Bowlby, 1980), se ha evidenciado que el maltrato afecta negativamente la calidad del apego (Baer \& Daly Martínez, 2006), siendo el maltrato una de las causas más importantes del desarrollo de un apego desorganizado (Carlson, 1998; Cicchetti \& Barnet, 1991; Van IJzendoorn, Schuengel \& BakermansKranenburg, 1999). No obstante lo anterior, son pocas las investigaciones que estudian el impacto específico del maltrato físico sobre las representaciones del apego infantil (Baer \& Daly Martínez, 2006; Toth et al., 2000).

Bowlby $(1969,1980)$ propone el término Modelo Interno Operante (M.I.O.) para describir las representaciones mentales que el bebé crea de sí mismo, de su figura de apego y de la relación entre ambos. Este modelo representacional se desarrolla tempranamente a partir de la internalización de pautas interaccionales entre la figura de apego y el niño (Bowlby, 1980). Si la figura de apego reconoce las necesidades de apego del niño y entrega una respuesta estable y predecible con afecto y protección, entonces el niño podrá crear un vínculo de apego seguro internalizándola como una representación de apego segura (Belsky \& Fearon, 2008 citados en Cassidy \& Shaver, 2008). Los M.I.O. permiten predecir e interpretar los comportamientos de la figura de apego y de los otros, también pueden guiar los pensamientos, emociones y conductas del sí mismo (Bretherton \& Munholland, 2008, citados en Cassidy \& Shaver, 2008), generando estrategias apropiadas para regular los comportamientos de apego (Kobak, Cole, Ferenz-Gillies, Fleming, \& Gamble, 1993). Frente a situaciones de amenaza, la estrategia primaria o segura corresponde a la puesta en acción de conductas para restablecer la cercanía con la figura de apego y ser calmado emocionalmente para luego explorar el entorno inmediato (Main, 1990). Las llamadas estrategias secundarias o inseguras se desarrollan cuando la figura de apego es insensible a las demandas de seguridad del niño, quien elabora patrones de comportamiento para enfrentar la amenaza sin perder el vínculo con la figura de apego. En el caso de la estrategia de desactivación, el niño desactiva su sistema de apego para evitar sentir el sufrimiento que genera el rechazo de la figura de apego (Howe, 2005; Main, 1990). Al contrario, en la estrategia de hiperactivación, el niño intensifica sus comportamientos de apego frente a respuestas inconsistentes por parte de los cuidadores para movilizar efectivamente la cercanía de éstos (Howe, 2005; Main, 1990). Cuando las respuestas del cuidador ante las necesidades de apego del niño son extremadamente incoherentes y/o maltratadoras, se hace imposible que el niño desarrolle una estrategia organizada de apego, activándose simultáneamente comportamientos contradictorios de acercamiento y escape ante la figura de apego (Lyons-Ruth, Bronfmang \& Atwood, 1999, citados en Solomon \& George, 1999; Main \& Hesse, 1990, citados en Greenberg, Cicchetti \& Cummings, 1990).

Los escasos estudios sobre los efectos del maltrato físico en las representaciones de apego indican que los niños, en sus narrativas, describen una representación negativa de la figura de apego como castigadora y rechazante, y una representación de sí mismo en términos de grandiosidad, con habilidades sobredimensionadas y grandes poderes (Toth et al., 2000). Por su parte Howe (2005) señala que los niños que experimentan maltrato físico tienen una representación 
de sí mismos negativa, de no ser dignos de ser protegidos ni cuidados. Si bien estos resultados indican que el maltrato físico afecta las representaciones de apego, hasta la fecha no se han encontrado estudios que den cuenta de este fenómeno en términos de las clasificaciones del apego tradicionalmente utilizada en la literatura de apego, esto es seguro, inseguro evitativo, inseguro ambivalente y desorganizado (Ainsworth, Blehar, Waters, \& Wall, 1978; Main $\&$ Solomon, 1986, citados en Brazelton \& Yogman, 1986).

Tomando en cuenta estos elementos se plantea como hipótesis que el maltrato físico afectará negativamente la calidad de las representaciones del apego favoreciendo la configuración de modelos internos operantes que orienten hacia estrategias de apego inseguras.

\section{Método}

\section{Participantes}

Fueron evaluados 36 niños de la Región del Maule con una edad promedio de 5,3 años. El grupo de estudio estuvo conformado por 8 niños diagnosticados preferentemente como víctimas de maltrato físico por centros de atención a la infancia especializados en maltrato infantil del Servicio Nacional de Menores del Gobierno de Chile (SENAME). Como lo señala la literatura, generalmente coexisten distintos tipos de maltrato (Cicchetti \& Toth, 2005), pudiendo presentar los niños del grupo de estudio maltrato psicológico y/o negligencia, pero en un grado menor en comparación al maltrato físico. El grupo de comparación estuvo compuesto por 28 niños sin sospecha de maltrato. Los niños de este grupo fueron reclutados en colegios de la Región del Maule donde, a juicio de los profesionales de los establecimientos, no serían víctimas de ningún tipo de maltrato. Ambos grupos pertenecen a un nivel socioeconómico bajo.

\section{Instrumentos}

Para evaluar el apego se utilizó la tarea de completación de historias de apego (Attachment Story Completion Task, A.S.C.T.) (Bretherton, Ridgeway \& Cassidy, 1990 citados en Greenberg, D. Cicchetti, \& E.M. Cummings), en su versión adaptada al español (Pierrehumbert et al., 2009). Este instrumento permite evaluar la calidad de las representaciones del apego en niños de 3 a 7 años, a través del análisis de sus narrativas. Se trata de 5 inicios de historias que los niños deben completar con la ayuda de unas figuras representando a una familia. Estas historias activarían las representaciones del apego de los niños. La actividad es filmada y tiene una duración aproximada de 30 minutos. El instrumento fue aplicado por psicólogas clínicas entrenadas en la A.S.C.T. Las narrativas de los niños fueron analizadas con el sistema de codificación A.S.C.T Q-sort (Miljkovitch, Pierrehumbert, Bretherton, \& Halfon, 2004). Este sistema permite calificar la calidad de las representaciones del apego en términos dimensionales, dando cuenta del privilegio por estrategias de seguridad, desactivación, hiperactivación y desorganización del apego. El evaluador, luego de visionar la actividad de juego, debe clasificar en 7 columnas los 65 ítems que dan cuenta de comportamientos durante la A.S.C.T. relacionados con el apego. Los puntajes obtenidos se correlacionan con cuatro prototipos de apego, lo que resulta en un puntaje para cada individuo de las dimensiones de seguridad, desactivación, hiperactividad y desorganización del apego. La codificación de cada video toma alrededor de 3 horas y fue realizada por dos psicólogas clínicas entrenadas en el sistema de codificación. Se trabajó con evaluadoras ciegas. El acuerdo interjuez para este estudio fue de 0.94, 0.95, 0.87 y 0.89 para cada dimensión del apego.

Para evaluar el maltrato físico se utilizó el Sistema Modificado de Clasificación de Maltrato (Modified Maltreatment Clasification System, M.M.C.S.) (English $\&$ the LONGSCAN Investigators, 1997). Se trata de un sistema que permite clasificar las diferentes manifestaciones del maltrato infantil en tipologías consensuadas: abuso sexual, maltrato físico y negligencia. La información acerca de las características del maltrato sufrido por un niño es recopilada a partir de los archivos de los centros de protección a la infancia que atienden el caso. Una vez obtenida la información, se contrasta con las categorías de maltrato del M.M.C.S. y se clasifica en una tipología. En este sistema la codificación de maltrato físico se aplica a los casos en que el cuidador o adulto responsable ocasiona lesiones físicas al niño, exceptuando aquellos casos en que las lesiones fueron accidentales. Este instrumento permite además consignar la gravedad del maltrato. Cada tipología cuenta con una escala, de 1 a 6 puntos, que permite definir la gravedad del maltrato en razón del daño producido al menor, donde un puntaje de 1 es leve (Ej: el cuidador da una palmada en la cara sin dejar huellas en la cara del niño) y 6 es grave con riesgo vital (Ej: el niño está en coma después de ser golpeado con un bate de béisbol por su cuidador). En este estudio la escala de gravedad se utilizó para descartar aquellos casos de coocurrencia de maltratos en que la negligencia o el maltrato psicológico fueran más graves que el maltrato físico. Si bien no se desconoce la importancia de considerar la gravedad del maltrato para comprender sus efectos en el desarrollo infantil, en el presente estudio los niños víctimas de maltrato físico son considerados en una categoría única sin distinción de gravedad. La codificación del maltrato fue realizada por psicólogas clínicas entrenadas en este sistema de clasificación.

\section{Procedimiento}

Para la realización de este estudio se contó con el patrocinio de SENAME y la colaboración de la Fundación CRATE, lo que permitió tener acceso a los centros de protección a la infancia especializados en el diagnóstico y 
tratamiento de niños maltratados. Posteriormente se tomó contacto con los equipos de los centros mencionados, junto con los cuales se seleccionó a posibles niños participantes. A través de los centros se contactó al adulto responsable del niño y se les invitó a participar del estudio. Los adultos que aceptaron participar firmaron un consentimiento informado que autorizaba la evaluación del niño y que se contactara al niño a su cargo. Luego se contactó a los niños, a quienes se les explicó el estudio y las características de la evaluación. Los niños accedían a participar a través de un consentimiento informado que se les leía, en el cual consignaban libre y voluntariamente que aceptaban realizar la actividad de juego. La firma de los consentimientos (adulto y niño) fue realizada en presencia de un profesional de la institución como testigo de fe. El tiempo de aplicación de la prueba fluctuó entre una a dos horas pon niño. Las evaluaciones se realizaron en los centros de atención de SENAME y en los colegios de los niños participantes. Los videos del juego de los niños fueron codificados con el sistema A.S.C.T. Q-sort. La codificación de cada video tomó un tiempo aproximado de 3 horas. De manera paralela se recopiló la información concerniente a las experiencias de maltrato de los menores en los archivos de los centros donde eran atendidos para luego codificarlo con el M.M.C.S. En el caso del grupo de comparación, los antecedentes de los menores fueron recopilados con los profesores y los apoderados de los niños. En todos los casos se resguardó el anonimato de los participantes.

\section{Resultados}

Para poder evidenciar el efecto del maltrato físico en la calidad de las representaciones del apego se utilizó ANOVA factorial. En el caso de que el efecto fuera significativo, se calculó el tamaño del efecto $d$ de Cohen (1988). En los análisis se consideró el efecto del maltrato físico para cada dimensión de apego evaluada con el A.S.C.T. Q-sort: segura, desactivada, hiperactivada y desorganizada.

A nivel descriptivo los resultados expuestos en la Tabla 1 muestran que, para el grupo de niños sin sospecha de maltrato, la media más alta corresponde a la dimensión de desactivación (52.7), luego les siguen la de desorganización (51.0), la de seguridad (48.7) y la de hiperactivación (47.0). En el caso del grupo de niños víctimas de maltrato físico, la estrategia con la media más alta es la de desorganización (58.5), le siguen la de hiperactivación (54.1), la de desactivación (52.7) y la de seguridad (46.5). Cabe destacar que las mayores diferencias entre grupos se aprecian en las dimensiones de hiperactivación y desorganización.

En la Tabla 2 se aprecia que el efecto del maltrato físico en la calidad del apego sólo es significativo para las dimensiones del apego que son hiperactivación y desorganización. El tamaño del efecto para la dimensión de hiperactivación es cercano al efecto medio y para la dimensión desorganización el efecto es grande (Cohen, 1988).

Tabla 1: Media y desviación típica de las cuatro dimensiones de apego en puntaje torganizadas por grupo: maltrato físico, sin maltrato.

\begin{tabular}{|c|c|c|c|}
\hline Dimensiones de apego en puntaje $\mathrm{t}$ & Grupo & Media & DT \\
\hline \multirow[t]{2}{*}{ Seguridad } & Maltrato físico & 46.5 & 8.8 \\
\hline & Sin maltrato & 48.7 & 11.8 \\
\hline \multirow[t]{2}{*}{ Desactivación } & Maltrato físico & 52.7 & 7.4 \\
\hline & Sin maltrato & 52.6 & 10.5 \\
\hline \multirow[t]{2}{*}{ Hiperactivación } & Maltrato físico & 54.1 & 8.3 \\
\hline & Sin maltrato & 47.0 & 8.8 \\
\hline \multirow[t]{2}{*}{ Desorganización } & Maltrato físico & 58.5 & 9 \\
\hline & Sin maltrato & 51.0 & 8.7 \\
\hline
\end{tabular}

Tabla 2: Análisis de la varianza para el efecto del maltrato físico en las cuatro dimensiones del apego en puntaje t (seguridad, desactivación, hiperactivación y desorganización) y tamaño del efecto (d).

\begin{tabular}{lcccc}
\hline Dimensiones de apego en puntaje $t$ & $g l$. & $F$ & $P$ & $d$ \\
\hline Seguridad & 1,34 &, 258 & .615 & .975 \\
Desactivación & 1,34 &, 001 & $.048^{*}$ & .4 medio \\
Hiperactivación & 1,34 & 4,195 & $.038^{*}$ & .9 grande \\
Desorganización & 1,34 & 4,639 & & \\
\hline
\end{tabular}

$* \mathrm{p}<.05$

Nota: Cohen (1988) considera un tamaño del efecto pequeño $=.2$, medio $=.5$ y grande $=.8$. 


\section{Discusión}

A nivel descriptivo se aprecia comparativamente que la dimensión de seguridad de apego es en general más baja que el promedio normalizado $(t=50)$ y que la diferencia entre ambos grupos no es considerable. Esto se puede deber a que ambos grupos pertenecen a un nivel socioeconómico bajo y rural, estando ambos grupos expuestos a una serie de factores de riesgo que afectan negativamente el nivel de seguridad del apego (Belsky \& Fearon, 2008). Esto es coherente con los hallazgos del meta análisis de Van IJzendoorn et al. (1999), que señala que en contextos de pobreza hay una subrepresentación del apego seguro en comparación con la población normal.

A partir de los análisis inferenciales se puede señalar que el maltrato físico tiene un efecto en la calidad de las representaciones del apego, comprobándose la hipótesis de trabajo, sin embargo sólo se ven afectadas las dimensiones de hiperactividad y desorganización del apego. Esto sugiere que los niños que sufren maltrato físico tienen mayor probabilidad de configurar estrategias de apego de tipo hiperactivado y desorganizado que los niños no maltratados. En el caso de la hiperactivación los niños intensifican sus comportamientos de apego para hacer frente a las inconsistencias parentales y obtener de parte de éstos una respuesta estable, configurándose un M.I.O. inseguro en el niño (Pierrehumbert, 2003). Bowlby (1980) plantea que estos niños podrían responder defensivamente a esta situación desconectando, a nivel cognitivo, la relación entre la emoción de malestar que genera la inconsistencia parental y la situación interpersonal real que la genera, aumentando la probabilidad de atribuir de manera errónea la emoción negativa experimentada a otra situación o hacia sí mismos. Esta hipótesis concuerda con lo señalado por Howe (2005) en relación a que los niños víctimas de maltrato físico desarrollan una representación de sí mismos negativa. Al mismo tiempo coincide con lo descrito por Toth et al. (2000), quienes indican que los niños presentan una imagen de sî mismos grandiosa, la cual se desprendería de narrativas donde los niños manifiestan que pueden golpear a los padres o a los hermanos. Según estos autores, correspondería a la configuración de una imagen compensatoria de alguien que puede enfrentar el daño recibido por una figura más fuerte y atemorizante. Se puede postular que esta imagen, al ser compensatoria, también daría cuenta de una imagen negativa de sí mismo en la base. Es importante señalar que en este caso la estrategia de apego sigue siendo organizada logrando mantener el vínculo con la figura de apego.

En el caso de la dimensión desorganización, los resultados indican que ésta también se ve afectada por el maltrato físico y que, comparativamente, el efecto es mayor que en la dimensión de hiperactivación. Los comportamientos de maltrato físico por parte de las figuras de apego hacen que éstas, en lugar de ser fuentes de protección y seguridad, sean figuras que generan terror e interacciones caóticas, lo que dificulta la posibilidad de establecer una estrategia de apego organizada (Carlson, Cicchetti, Barnett, \& Braunwald, 1989; Pierrehumbert, 2003; Solomon \& George, 1999). Frente a una figura de apego maltratadora, los niños responden con comportamientos contradictorios, de acercamiento, fuga, miedo, congelamiento, entre otras (Main \& Hesse, 1990). Los menores permanecen en un estado de hiperactivación emocional, apreciándose una pérdida de control del comportamiento, pasando de conductas de desesperanza a conductas hostiles (Howe, 2005). En términos defensivos, estos niños responden segregando el sistema representacional principal, creando múltiples sistemas representacionales del sí mismo segregados e incoherentes, lo que explicaría las conductas erráticas del menor (Liotti, 1999). Esto es coherente con las narrativas de apego de los niños, ya que durante la A.S.C.T., sus historias se caracterizan por ser incoherentes y caóticas, evidenciándose agresiones, destrucción y muerte, sin lograr desenlaces positivos (Miljkovitch et al., 2004).

Estos resultados son de gran relevancia ya que se ha establecido que los niños que desarrollan un apego desorganizado tienen más probabilidades de desarrollar psicopatología a corto, mediano y largo plazo (Baer \& Daly Martínez, 2006; Liotti, 2004; Lyon-Ruth \& Jacobvitz, 2008).

Los resultados de este estudio ponen en evidencia los efectos negativos del maltrato físico en la configuración de las representaciones del apego, mostrando la necesidad de contar con políticas públicas que apunten al tratamiento psicológico especializado de niños y niñas víctimas de maltrato. Esto no sólo para que ayuden al niño a elaborar el trauma de la situación de maltrato, sino también para prevenir el desarrollo de psicopatología posterior. Algunos autores (Crowell \& Waters, 2005, citados en Grossmann, Grossmann \& Waters, 2005; Sroufe, Egeland, Carlson \& Collins, 2005, citados en Grossmann, Grossmann \& Waters 2005), señalan que los M.I.O. pueden ser modificados hacia el polo de la seguridad, por lo que contar con psicoterapeutas que trabajen desde la teoría del apego permitiría realizar intervenciones que favorezcan que las representaciones de los niños víctimas de maltrato evolucionen hacia un apego seguro. Junto a lo anterior se deben destinar recursos para desarrollar programas de prevención del maltrato sustentables en el tiempo, así como políticas claras y efectivas en lo que se refiere a la promoción de la salud infantil, específicamente programas que favorezcan aquellos factores que fomentan un apego seguro entre los padres y sus hijos, previniendo así la aparición de maltrato infantil.

En términos de los límites de este estudio, cabe señalar que los datos presentados son resultados preliminares de un estudio más amplio, por lo que la generalización de los mismos debe ser tomada con cautela, sobre todo considerando el tamaño de esta muestra. Se sugiere para futuras investigaciones ampliar el número de participantes 
y considerar en los análisis otros factores relacionados con la configuración del maltrato que afectan el desarrollo de los niños, como por ejemplo la gravedad del maltrato, la periodicidad, la frecuencia, el vínculo con el perpetrador, la coocurrencia de otros tipos de maltrato, etc., que podrían afectar la configuración de las representaciones de apego infantil. A pesar de dichas limitaciones, el presente estudio contribuye a evidenciar el impacto devastador del maltrato sobre el desarrollo infantil desde un área de la psicología poco estudiada hasta el momento, como son las representaciones de apego infantil, y con una muestra chilena, permitiendo dar sustento a intervenciones que se adecuen a nuestra realidad nacional.

\section{Referencias}

Ainsworth, M. D. S., Blehar, M. C., Waters, E. \& Wall, S. (1978). Patterns of attachment: A psychological study of the Strange Situation. Hillsdale, NJ: Erlbaum.

Baer, J. C. \& Daly Martínez, C. (2006). Child maltreatment and insecure attachment: A meta-analysis. Journal of Reproductive and Infant Psychology, 24, $187-197$.

Barnett, D., Manly, J. T. \& Cicchetti, D. (1993). Defining child maltreatment: The interface between policy and research. In D. Cicchetti \& S.L. Toth (Eds.), Advances in applied developmental psychology: Child abuse, child development and social policy (pp. 7-73). Norwood, NJ: Ablex Publishing Corp.

Belsky, J. \& Fearon, P. (2008). Precursors of attachment security. In J. Cassidy \& P.R. Shaver (Eds.), Handbook of attachment: Theory, research, and clinical applications (pp. 295-316). New York: Guilford.

Bowlby, J. (1969). Attachment and loss: Vol. 1. Attachment. New York: Basic Books.

Bowlby, J. (1980). Attachment and loss: Vol. 3. Loss: Sadness and depression. New York: Basic Books.

Bretherton, I. \& Munholland, K. A. (2008). Internal working models in attachment relationships: Elaborating a central construct in attachment theory. En J. Cassidy \& P. R. Shaver (Eds.), Handbook of attachment: Theory, research, and clinical applications (pp. 102-127). New York: Guilford Press.

Bretherton, I., Ridgeway, D. \& Cassidy, J. (1990). Assessing internal working models of the attachment relationship. An attachment story completion task for 3-year-olds. En M. Greenberg, D. Cicchetti \& E.M. Cummings (Eds), Attachment during the preschool years (pp. 273-308). Chicago: University of Chicago Press.

Carlson, E. A. (1998). A prospective longitudinal study of disorganized/ disoriented attachment. Child Development, 69, 1107-1128.

Carlson, V., Cicchetti, D., Barnett, D. \& Braunwald, K. (1989). Disorganized/disoriented attachment relationships in maltreated infants. Developmental psychology, 25, 525-531.

Cicchetti, D. \& Barnett, D. (1991). Attachment organization in maltreated preschoolers. Development and Psychopathology, 3, 397-411.

Cicchetti, D. \& Toth, S. L. (2005). Child maltreatment. Annual Review of Clinical Psychology, 1, 409-438.

Cohen, J. (1988). Statistical power analysis for the behavioral sciences. Hillsdale, NJ: Lawrence Earlbaum Associates.

Crowell, J. \& Waters, E. (2005). Attachment representations, secure-base behaviour, and the evolution of adult relationships: The Stony Brook adult relationship project. En K. Grossmann, K. Grossmann \& E. Waters (Eds.), Attachment from infancy to adulthood: The major longitudinal studies (pp. 223-244). New York: The Guildford Press.

Elder, G.H., Van Nguyen, T. \& Caspi, A. (1985). Linking family hardship to children's lives. Child Development, 56, 361-375.

English, D. J. \& the LONGSCAN Investigators (1997). Modified maltreatment classification system (MMCS). Extraído el 11 de enero, 2011, disponible en http://www.iprc.unc.edu/longscan/pages/maltx/mmcs/ LONGSCAN\%20MMCS\%20Coding.pdf

Hodges, S. \& Steele M. (2000). Effects of abuse on attachment representations: Narrative assessments of abused children. Journal of Child psychotherapy, 26, 433-455.

Howe, D. (2005). Child abuse and neglect: Attachment, development and intervention. New York: Palgrave Macmillan.

Kobak, R. R., Cole, H., Ferenz-Gillies, R., Fleming, W. \& Gamble, W. (1993). Attachment and emotion regulation during mother-teen problem-solving: A control theory analysis. Child development, 64, 231-245.

Liotti, G. (1999). Disorganized attachment as a model for the understanding of dissociative psychopathology. En J. Solomon \& C. George (Eds.), Attachment Disorganization (pp. 291-317). New York: Guilford Press.

Liotti, G. (2004). Trauma, dissociation and discorganized attachment: Three strands of a single braid. Psychotherapy: Theory, Research, Practice, Training, 41, 472-486.

Lyons-Ruth, K., Bronfman, E. \& Atwood, G. (1999). A relational diathesis model of hostile-helpless states of mind: Expressions in mother-infant interaction. En J. Solomon \& C. George (Eds.), Attachment disorganization. (pp. 33-70). New York: Guilford Press.

Lyon-Ruth, K \& Jacobvitz, D. (2008). Attachment disorganization: Genetic factors, parenting contexts, and developmental transformation from infancy to adulthood. En J. Cassidy \& P. R. Shaver (Eds.), Handbook of attachment: Theory, research, and clinical applications (pp. 666-697). New York: Guilford Press.

McLoyd, V.C. (1990). The impact of economic hardship on black families and children: Psychological distress, parenting, and socioemotional development. Child Development, 61, 311-346.

Main, M. (1990). Cross-cultural studies of attachment organization: Recent studies, changing methodologies, and the concept of conditional strategies. Human Development, 33, 48-61.

Main, M. \& Hesse, E. (1990). Parents' unresolved traumatic experiences are related to infant disorganized attachment status: Is frightening and/or frightened parental behavior the linking mechanism? En M. T. Greenberg, D. Cicchetti \& E. M. Cummings (Eds.), Attachment in the preschool years (pp. 121-160). Chicago: University of Chicago Press.

Main, M. \& Solomon, J. (1986). Discovery of a new, insecure disorganized/ disoriented attachment pattern. En T. B. Brazelton \& M. Yogman (Eds.), Affective development in infancy (pp. 95-124). Norwood, NJ: Ablex.

Miljkovitch, R., Pierrehumbert, B., Bretherton, I. \& Halfon, O. (2004). Associations between parental and child attachment representations. Attachment \& Human Development, 6, 305-325.

Pierrehumbert, B. (2003). Le premier lien: Théorie de l'attachement. París: Odile Jacob.

Solomon, J. \& George, C. (1999). The place of disorganization in attachment theory.Attachment disorganization. En J. Solomon \& C. George (Eds.), Attachment disorganization. (pp. 3-32). New York: Guilford Press.

Pierrehumbert, B., Santelices, M.P., Ibáñez, M., Alberdi, M., Ongari, B., Roskam, I., Stievenard, M., Spencer, R., Fresno Rodríguez, A. \& Borghini, A. (2009). Gender and attachment representations in the preschool years: comparisons between five countries. Journal of Cross-Cultural Psychology, 40, 543-566.

Sroufe, L.A., Egeland, B., Carlson, E. \& Collins W.A. (2005). Placing early attachment experiences in developmental context. The Minnesota longitudinal study. En K. Grossmann, K. Grossmann \& E. Waters (Eds.), Attachment from infancy to adulthood: The major longitudinal studies (pp. 48-70). New York: The Guildford Press.

Toth, S, L., Cicchetti, D., Macfie, J., Maughan, A. \& Vanmeenen, K. (2000). Narrative representations of caregivers and self in maltreated pre-schoolers. Attachment \& Human Development, 2, 271-305.

UNICEF (2008). Maltrato infantil y relaciones familiares en Chile, análisis comparativo 1994 - 2000 - 2006. Santiago: Andros Impresores.

van IJzendoorn, M. H., Schuengel, C. \& Bakermans-Kranenburg, M. J. (1999). Disorganized attachment in early childhood: Meta-analysis of precursors, concomitants, and sequelae. Development and Psychopathology, 11, 225-249. 


\title{
Regulación de la Agresividad Entre Preescolares Mediante el Entrenamiento a Madres y Profesoras*
}

\section{Regulation of The Aggressiveness Among Pre-School Children by The Training of Mothers and Teachers}

\author{
Cristóbal Guerra \\ Universidad Santo Tomás, Chile \\ Verónica Fredes \\ Universidad del Mar, Chile
}

\author{
María de los Ángeles Campaña \\ Universidad del Mar, Chile \\ Lisette Gutiérrez \\ Universidad del Mar, Chile
}

$\&$

\author{
Hugo Plaza \\ Universidad del Mar, Chile
}

(Rec: 20 de Enero de 2010 / Acep: 14 de Septiembre de 2011)

\begin{abstract}
Resumen
El objetivo de la investigación fue evaluar la efectividad de una intervención dirigida a disminuir la frecuencia de conductas agresivas en preescolares mediante el entrenamiento a madres y profesoras. Se diseñó e implementó un programa de entrenamiento a madres y profesoras tendiente a modificar sus creencias irracionales referidas a la crianza de los niños y a capacitarlas en una serie de procedimientos de manejo conductual infantil. El diseño empleado para evaluar la efectividad del tratamiento fue el de línea base múltiple. Se analizan los resultados de la intervención en base a criterios de significación clínica y estadística. La intervención fue exitosa ya que las madres y profesoras disminuyeron sus creencias irracionales y mejoraron sus habilidades de manejo conductual para hacer frente a la agresividad de los niños. De esta manera los niños disminuyeron la frecuencia del comportamiento agresivo verbal, físico y oposicionista tanto en el contexto familiar como escolar.
\end{abstract}

Palabras clave: Agresividad, preescolares, entrenamiento a padres, intervención en contexto escolar, tratamientos efectivos.

\begin{abstract}
The aim of this research was to evaluate the effectiveness of an intervention directed to reduce the frequency of aggressive behavior among pre- school children, by training their mothers and teachers. A training program for mothers and teachers was designed and implemented, in order to modify their irrational beliefs referred to the guidance of kids and to teach them appropriate procedures for modification in the children's behavior. The design for the assessment of an effective treatment implementation was the so called multiple baseline design in terms of clinical and statistical meaning. The intervention was successful since the mothers and teachers diminished his irrational beliefs and improved their skills of behavior modification when tackling the aggressiveness of the toddlers. On the other hand, the children also diminished the frequency of aggressive verbal, physical and opponent behavior both within the family and school contexts.
\end{abstract}

Key words: Aggressiveness, pre- school children, parents' training, intervention in school context, effective treatments. 


\section{Introducción}

La agresividad entre escolares es un fenómeno de creciente interés internacional debido a su alta incidencia en todo el mundo (Trautmann, 2008). Esto se ve reflejado en distintos estudios de prevalencia y en estudios que buscan delimitar sus consecuencias.

En Chile el 45,5\% de los estudiantes de colegios públicos han observado episodios de violencia física entre sus pares (Zerón, 2002), mientras que en España el 48\% de los estudiantes de secundaria han sido agredidos por sus pares (Gutiérrez, Barrios; de Dios, Montero \& del Barrio, 2008). Por su parte, un estudio de prevalencia realizado en 25 países revela que la agresividad escolar varía entre un $9 \%$ en Suecia y un $54 \%$ en Lituania (Nansel, Craig, Overpeck, Saluda \& Ruan, 2004).

Respecto a las consecuencias de la agresividad entre escolares, se ha documentado que un comportamiento excesivamente agresivo en la infancia se asocia al fracaso académico (Lozano \& García, 2000), predice la agresividad durante la adolescencia y la edad adulta (Kellam, Rebok, Ialongo \& Mayer, 1994) y predice otras patologías psicológicas durante la edad adulta (De la Barra, Toledo \& Rodríguez, 2003). Por otro lado, los trastornos depresivos y ansiosos, el ausentismo escolar, el deterioro en el rendimiento académico y los desajustes psicosociales en la adultez son indicadores comúnmente visibles en las víctimas de las agresiones (Trautmann, 2008).

La psicología ha aportado en la generación de intervenciones destinadas a disminuir la agresividad entre escolares y a tratar sus consecuencias. En esta línea, Trianes \& Fernández-Figarés (2001) desarrollaron un programa de intervención tendiente a la mejora del clima escolar en adolescentes mediante el entrenamiento en estrategias de resolución de conflictos. Además hay una serie de autores que han implementado, con éxito, programas de modificación conductual para regular las conductas disruptivas y agresivas en escolares. Por ejemplo, Aguilar \& Navarro (2008) utilizaron la economía de fichas y el contrato de contingencias en alumnos de primaria; Pérez, Fernández, Rodríguez \& De la Barra (2005) aplicaron un programa de contingencias llamado "el juego del buen comportamiento", también en niños de primaria; Livacic-Rojas, Espinoza \& Ugalde (2004) aplicaron un programa de habilidades sociales a adolescentes; mientras que Corsi, Barrera, Flores, Perivancich \& Guerra (2009) utilizaron la economía de fichas, la alabanza verbal, las instrucciones y el costo de respuesta en estudiantes de secundaria.

Todos los trabajos recién descritos han sido dirigidos a escolares de distintas edades, pero ninguno de ellos aborda el fenómeno de la agresividad en preescolares. Esto es inquietante ya que se han descrito indicadores del comportamiento antisocial y de agresividad injustificada a partir de los 3 años, por lo que se hace necesario realizar investigaciones con esta población (Benítez \& Justicia, 2006; Ortega \& Monks, 2005).

Motivados por lo anterior, y considerando que hoy en día el ingreso al sistema educativo en muchos países comienza en el jardín de infantes, es que ésta investigación pretendió diseñar, implementar y evaluar una intervención tendiente a la disminución de la frecuencia de la conducta agresiva en preescolares. Además, las intervenciones en la etapa preescolar generan un alto impacto ya que, como señalan Urzúa, Ramos, Alday \& Alquinta (2010), los preescolares son altamente suceptibles a la influencia ambiental debido al proceso de maduración neuropsicológica.

La intervención se sustenta en el entrenamiento a madres y profesoras ya que está documentada la relación entre los estilos de crianza, caracterizados por la difusión de normas y por la utilización excesiva del castigo, con la conducta desadaptativa en niños (Benítez \& Justicia, 2006; Mestre, Tur, Samper, Nácher \& Cortés, 2007; Schwartz, Dodge, Pettit \& Bates, 1997). Por otro lado, diversos estudios reportan resultados beneficiosos de realizar intervenciones que incluyan a padres (Corsi, Guerra \& Plaza, 2007; Rey, 2006) y a profesores (Corsi, Barrera, Flores, Perivancich, \& Guerra 2009, Trautmann, 2008).

\section{Método}

\section{Participantes}

Participaron de este estudio un grupo de 3 preescolares varones, con sus respectivas madres y profesoras.

El rango de edad de los niños fluctuó entre los 3 años con 6 meses y los 4 años con 6 meses. Todos los niños pertenecían a familias de bajos recursos que residían y estudiaban en la ciudad de Quilpué, en Chile. Los tres niños presentaban una alta frecuencia de conductas agresivas tanto en su casa como en el contexto escolar. Específicamente presentaban una alta frecuencia de conducta agresiva verbal (gritos, burlas, garabatos e insultos hacia sus pares y adultos), conducta agresiva física (golpes de puño y pie, mordiscos, lanzamiento de objetos hacia sus pares y adultos) y oposicionismo (lanzar objetos al suelo, ejecutar pataletas, prohibir acciones a otros y negarse a seguir las instrucciones dadas por madres y profesoras).

El rango de edad de las madres y profesoras fluctuó entre los 22 y 48 años. Tanto madres como profesoras presentaban una serie de creencias irracionales referidas a la crianza de los niños (ej. "nada de lo que haga va a hacer cambiar a este niño", "si no dejo que el niño haga lo que quiera seré una mala madre/profesora"). Además presentaban dificultades para abordar las conductas agresivas de los niños (instrucciones poco claras, refuerzo de conductas agresivas, ausencia de refuerzo ante las conductas positivas de los niños, inconsistencia en la aplicación de normas, maltrato físico y verbal hacia los niños). 
Al finalizar el programa de intervención se esperó que las madres y profesoras disminuyeran la frecuencia de sus creencias irracionales referidas a la crianza de sus hijos o alumnos respectivamente. Además se esperó que las madres y profesoras mejoraran sus habilidades de manejo conductual, específicamente que fueran capaces de dar instrucciones de forma adecuada (asegurándose que el niño esté prestando atención, dando la instrucción en un tono firme y describiendo claramente la conducta solicitada al niño) y de administrar técnicas de refuerzo y castigo de forma adecuada (reforzando conductas prosociales y castigando las conductas agresivas de forma consistente y contingente a la emisión de la conducta por parte del niño). Finalmente, se esperó que los niños disminuyeran la frecuencia de la conducta agresiva verbal, física y oposicionista.

\section{Instrumentos}

1. Escala de creencias irracionales para madres y profesoras (Campaña, Fredes \& Gutiérrez, 2008). Corresponde a una escala de autorregistro de 12 ítems, divididos en 3 subescalas de 4 ítems cada una. Cada subescala mide un tipo de creencia irracional asociada a la crianza de los niños; tremendismo (ejemplos: castigar al niño es algo terrible, es imposible controlar a este niño), nosoportitis (ejemplos: no soporto ver al niño llorar cuando lo disciplino, no soporto no lograr lo que me propongo con el niño), y autocondena (ejemplos: el niño no me va a querer si le pongo reglas, castigarlo me hace una mala madre/profesora).

Las participantes debieron indicar con qué frecuencia experimentaban la creencia medida por cada ítem. La escala de frecuencias incluye los siguientes valores: $0=$ nunca, 1 $=\mathrm{a}$ veces, $2=$ siempre. Para interpretar los resultados se sumaron los puntajes de los reactivos correspondientes a cada subescala, estos puntajes van de 0 a 8 (a mayor puntaje mayor frecuencia de las creencias irracionales).

2. Hojas de registro para las conductas de niños, madres y profesoras: Se utilizaron hojas de registro de la frecuencia con que cada conducta objetivo era emitida por los participantes. Las hojas de registro estaban divididas en celdas que representaban intervalos de 5 minutos. De este modo, cada sesión de observación de 45 minutos fue representada en una hoja dividida en 9 intervalos. Se marcaba la presencia de la conducta objetivo cuando ésta se presentaba, a lo menos una vez, dentro del intervalo de tiempo correspondiente. Esta forma de registro se denomina muestreo temporal de intervalo parcial (Cooper, Heron \& Heward, 2006).

Posteriormente se calculó el porcentaje de intervalos en que estuvo presente cada conducta objetivo y se graficó su frecuencia. Para asegurar la fiabilidad de las observaciones, se utilizó el método de observadores independientes durante todas las mediciones. Los observadores independientes alcanzaron un $88 \%$ de acuerdo, lo que es adecuado según los criterios de Forehand \& Mc Mahon (1981).

\section{Diseño}

Se utilizó un diseño de línea base múltiple tanto para evaluar los cambios en las creencias de las madres y profesoras, como para evaluar los cambios en las conductas de las madres, profesores y niños (medidas en cada sesión individual). Las fases de intervención consideradas en este diseño fueron:

1. Línea Base: En esta fase se registró el puntaje obtenido en la escala de creencias irracionales por madres y profesoras previo a la implementación del tratamiento. Además se registró el porcentaje del tiempo en que madres y profesoras daban instrucciones adecuadamente a los niños y administraban contingencias adecuadamente ante la conducta de los niños, previo al inicio del tratamiento. Finalmente se registró el porcentaje del tiempo en que los niños emitían conductas agresivas verbales, físicas y oposicionistas antes de iniciado el tratamiento. Las sesiones de línea base fueron 3 para el primer niño (también para su madre y profesora), 5 para el segundo niño (también para su madre y profesora) y 7 para el tercer niño (también para su madre y profesora)

2. Fase de tratamiento: En esta fase se registró el puntaje obtenido por madres y profesoras en la escala de creencias irracionales, las conductas objetivo de madres y profesoras y las conductas objetivo de los niños, una vez iniciada la intervención. Todos los participantes recibieron 7 sesiones de tratamiento individual que se realizaron en un período de 6 semanas. Además las madres y profesoras (por separado) participaron de un taller grupal (de 3 sesiones de 2 horas cada una), realizadas en las dependencias del establecimiento educacional.

3. Fase de seguimiento: En esta fase se registraron las cogniciones y conductas objetivo de los participantes después de quince días y después de un mes del término del tratamiento.

\section{Procedimiento}

Se realizó un proceso de evaluación conductual tendiente a identificar las conductas objetivo y las variables asociadas a su mantención. Se estableció que las creencias irracionales de las madres y profesoras se asociaban a sus dificultutades en el manejo conductual de los niños. A su vez, se consideró que las conductas agresivas de los niños eran mantenidas por este manejo conductual inadecuado de madres y profesoras. En la figura 1 se presenta la relación entre las variables recién descritas.

Luego, para lograr los cambios cognitivo-conductuales en madres y profesoras se utilizaron dos modalidades de intervención: La primera fue un taller grupal dirigido a madres y profesoras. La segunda fue un conjunto de sesiones individuales con madres y profesoras, en presencia de los niños, realizadas en sus propias casas y en el colegio. 


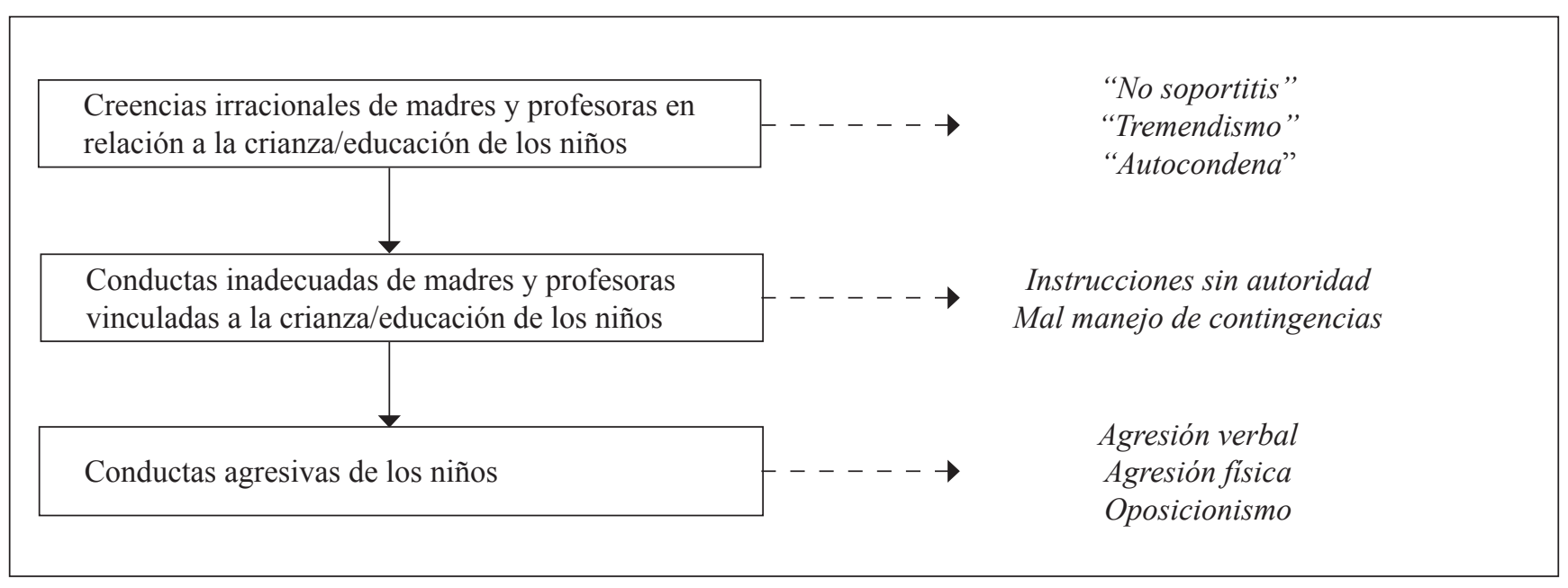

Figura 1: Evaluación conductual: a profesoras, madres y niños

Durante las sesiones grupales, madres y profesoras fueron entrenadas en técnicas de modificación conductual para aplicar con los niños y se trabajó en la modificación de sus creencias irracionales. Para modificar las creencias irracionales se utilizó autorregistro y debate cognitivo (para ver una descripción detallada de estas técnicas consultar Ellis, 1999). Luego, para capacitarlas en las técnicas de modificación conductual se utilizó la psicoeducación (donde los investigadores exponían, en términos simples, los fundamentos del análisis conductual aplicado), el modelado (mediante juego de roles los investigadores mostraban la forma de aplicar las técnicas), la imaginería (donde madres y profesoras imaginaban los efectos favorables de las técnicas a aplicar) y el entrenamiento en autoinstrucciones (de forma que madres y profesoras pudieran guiar la propia ejecución de las técnicas aprendidas). Para una descripción detallada de estas técnicas consultar Caballo (1998).

Paralelamente en las sesiones de entrenamiento individual se utilizó el ensayo de conducta, donde madres y profesoras debían aplicar directamente a los niños las técnicas de modificación conductual aprendidas en las sesiones del taller grupal. El rol de los investigadores incluyó moldear la conducta de madres y profesoras, es decir, entregar retroalimentación y refuerzos verbales a las participantes de manera de ayudarlas a mejorar gradualmente en la ejecución de las técnicas. Para una descripción detallada de estas técnicas consultar Caballo (1998).

Mediante estas dos modalidades de entrenamiento las madres y profesoras fueron entrenadas en una serie de técnicas de modificación conductual para aplicar con los niños. Las profesoras fueron entrenadas en la forma de aplicar instrucciones y contingencias adecuadas a la conducta de los niños en el contexto escolar. Específicamente las profesoras fueron entrenadas en técnicas de refuerzo verbal, economía de fichas, guía manual y costo de respuesta. Paralelamente, en el hogar de los niños, las madres fueron entrenadas en las mismas técnicas, con la diferencia que en lugar de la economía de fichas se incorporó el tiempo fuera de refuerzo (de 5 minutos) y la restricción física pasiva. Para una revisión más detallada de las técnicas aplicadas por profesoras y madres revisar Foxx, 1982a/b). La figura 2 muestra cómo se integran las distintas modalidades y contextos de la intervención.

Para evaluar la significación clínica de los cambios generados por el tratamiento se llevaron los datos tomados de las conductas y cogniciones de cada uno de los participantes, en cada una de las sesiones individuales, a un gráfico de línea base múltiple de 3 sujetos. Finalmente, para evaluar la significación estadística de los cambios generados por el tratamiento se realizaron análisis con la prueba $\mathrm{F}$ de ANOVA para cada una de las variables cognitivas y conductuales medidas. Además se evaluaron las diferencias entre medias mediante contraste pos hoc DMS, igualdad de varianza de error (contrastes de Levene), la potencia de la prueba y el tamaño del efecto. Por tratarse de un diseño de línea base múltiple, no se registró el mismo número de sesiones de línea base para todos los participantes (generando datos perdidos en las sesiones de línea base de los niños 1 y 2 , sus madres y profesoras). Para realizar los análisis estadísticos se reemplazaron los datos perdidos por la media obtenida por cada uno de estos sujetos en la fase de línea base. Para la realización de los análisis estadísticos se utilizó el programa SPSS 11.0.

\section{Resultados}

\section{Observación directa y análisis visual}

Dado que esta intervención se realizó paralelamente en el contexto escolar y familiar, los resultados se expresan de esa manera. 


\section{Técnicas de entrenamiento a profesoras}

Utilizadas en sesiones grupales:

- Psicoeducación

- Autorregistro de creencias irracionales

Debate cognitivo

Imaginería

Entrenamiento en auto-instrucciones

- Modelado
Utilizadas en la sala de clases y recreo:

- Ensayo de conducta

- Moldeamiento

\section{Efectos del entrenamiento a las profesoras}

- Disminuyen creencias irracionales (tremendismo, autocondena y nosoportitis)

- Mejoran desempeño en la entrega de instrucciones a los niños

Mejoran el desempeño en la entrega de contingencias de refuerzo y castigo a los niños (alabanza verbal, guía manual, entrega y retiro de fichas)

\section{Cambio de conductas de los niños en contexto escolar}

- Disminuyen conductas oposicionistas

- Disminuyen conductas de agresión verbal

- Disminuyen conductas de agresión física

Cambio de conductas de los niños en contexto familiar

Disminuyen creencias irracionales (tremendismo, autocondena y nosoportitis)

- Mejoran desempeño en la entrega de instrucciones a los niños

- Mejoran el desempeño en la entrega de contingencias de refuerzo y castigo a los niños (alabanza verbal, guía manual, tiempo fuera, restricción física pasiva)

\section{Efectos del entrenamiento a las madres}

\begin{tabular}{|l|l|}
\hline Utilizadas en sesiones grupales: & $\begin{array}{l}\text { Utilizadas en sesiones individuales en la casa: } \\
-\quad \text { Ensayo de conducta } \\
-\quad \text { Psicoeducación }\end{array}$ \\
- $\quad$ Autorregistro de creencias irracionales & Moldeamiento \\
- Debate cognitivo & \\
$-\quad$ Imaginería & \\
$-\quad$ Entrenamiento en auto-instrucciones & \\
\hline \multicolumn{2}{|c|}{ Técnicas de entrenamiento a las madres } \\
\hline
\end{tabular}

Figura 2: Resumen del programa de intervención 


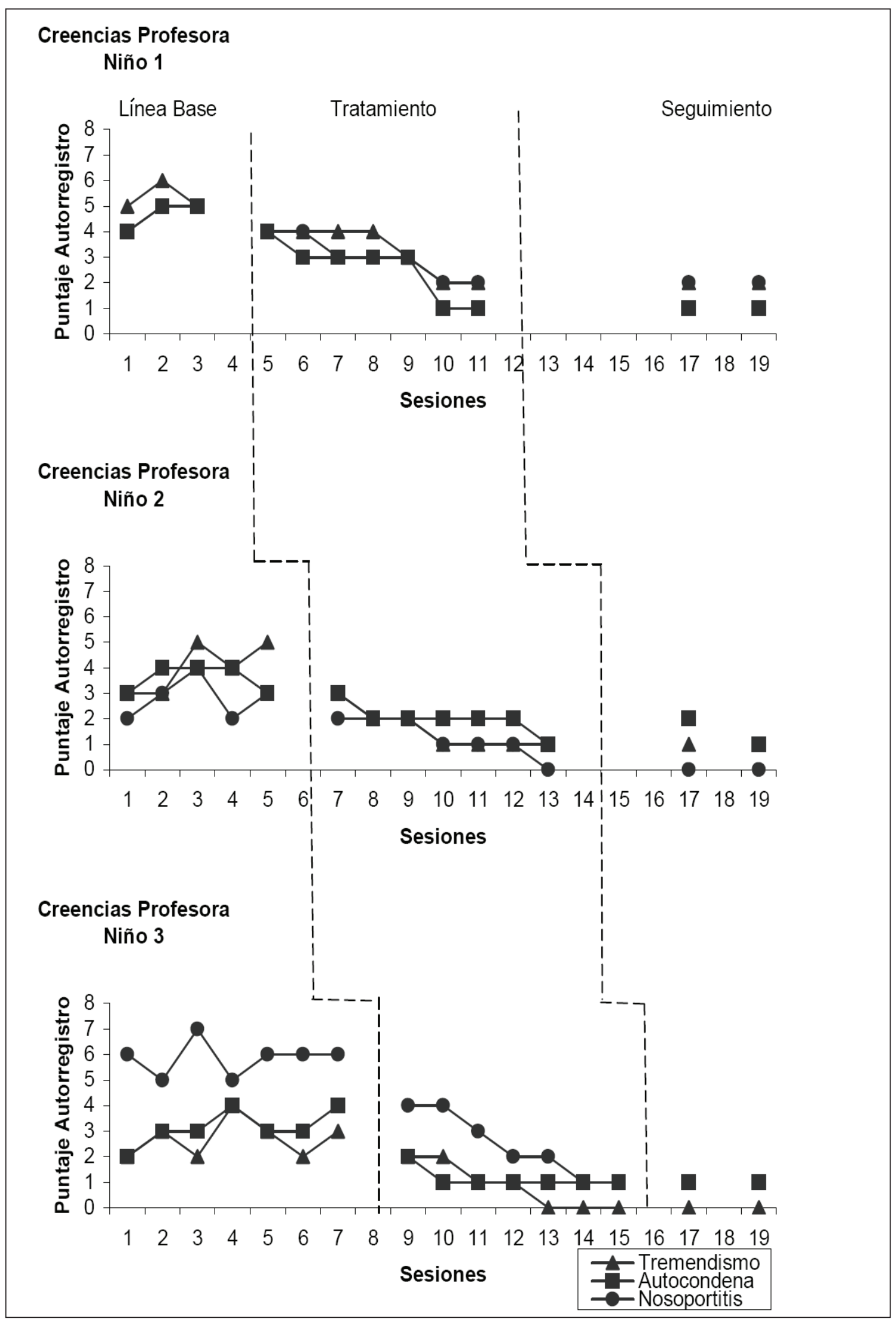

Figura 3: Cambios cognitivos en las profesoras en contexto escolar 


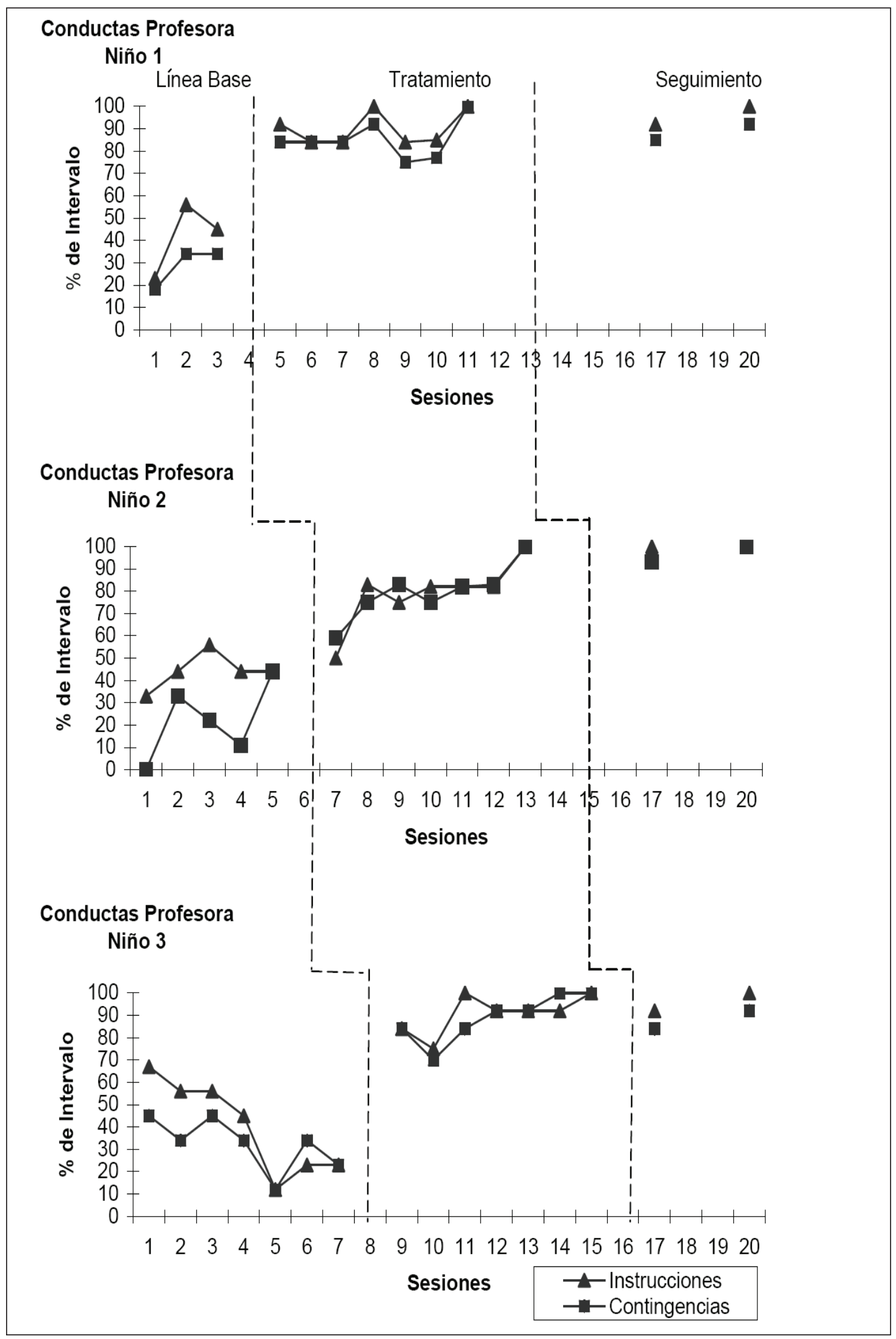

Figura 4: Cambios Conductuales en las profesoras en contexto escolar 


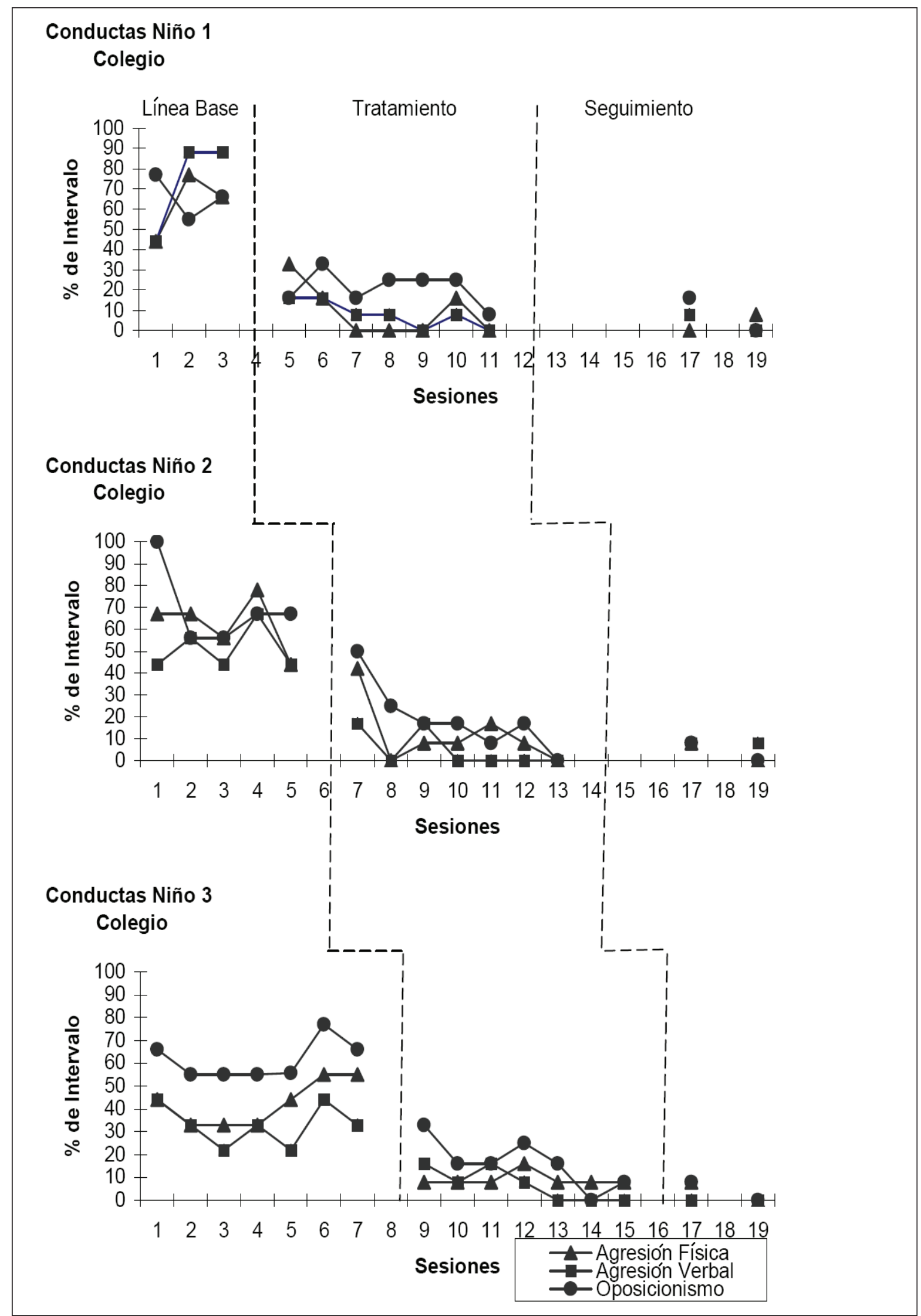

Figura 5: Cambios Conductuales en los niños en contexto escolar 
La figura 3, 4 y 5 representa los cambios cognitivos y conductuales en el contexto escolar. En la fase de línea base las tres profesoras presentaban una alta frecuencia de creencias irracionales del tipo nosoportitis (rango entre 2 y 7 puntos), tremendismo (rango entre 2 y 6 puntos) y autocondena (rango entre 2 y 5 puntos), vinculadas al comportamiento de los niños. Esto se asociaba a un bajo rendimiento en la entrega de instrucciones adecuadas a los niños (entre el 23\% y 56\% del tiempo), así como un bajo rendimiento en la aplicación adecuada de contingencias de refuerzo y castigo a los mismos (entre el $0 \%$ y $45 \%$ del tiempo). Consecuentemente, los tres niños presentaban, durante la línea base, una alta frecuencia de conducta agresiva verbal (entre el 22\% y $88 \%$ del tiempo), física (entre el 33\% y $78 \%$ del tiempo) y oposicionista (entre el $55 \%$ y $100 \%$ del tiempo). Una vez iniciada la intervención, las creencias irracionales de las tres profesoras fueron disminuyendo su frecuencia gradualmente hasta llegar a niveles mínimos (2 puntos o menos en todos los casos). La disminución de la frecuencia de las creencias irracionales de las profesoras coincidió con la mejora en su desempeño para hacer frente a la conducta de los niños. Específicamente, durante la intervención las profesoras lograron aplicar de forma adecuada instrucciones verbales a sus alumnos, así como contingencias de refuerzo y castigo adecuadas (alabanza verbal, entrega de fichas, guía manual y costo de respuesta). El rendimiento adecuado en ambas conductas objetivo llegó al 100\% del tiempo, en las tres profesoras, durante la última sesión de intervención. Tal como se esperaba, una vez que las profesoras comenzaron a mejorar su desempeño en la administración de instrucciones y contingencias, los niños presentaron cambios conductuales en el sentido deseado, al disminuir la frecuencia del comportamiento agresivo físico (entre el $0 \%$ y $8 \%$ en la última sesión de intervención), verbal (llegando al $0 \%$ en la última sesión de intervención en los tres niños) y oposicionista (entre el $0 \%$ y $8 \%$ en la última sesión de intervención). Estos resultados se mantuvieron durante la fase de seguimiento realizado a los 15 días y al mes después de finalizada la intervención, lo que da cuenta de la efectividad del tratamiento en el contexto escolar.

En el contexto familiar, la intervención también fue exitosa ya que, como se puede ver en las figura 6,7 y 8 las madres de los tres niños presentaban una alta frecuencia de las creencias irracionales medidas en línea base: nosoportitis (rango entre 2 y 8 puntos), tremendismo (rango entre 5 y 8 puntos) y autocondena (rango entre 2 y 8 puntos). Tal como en el caso de las profesoras, la existencia de creencias irracionales se asociaba al bajo rendimiento en la entrega de instrucciones (entre el $0 \%$ y $67 \%$ del tiempo) y contingencias (entre el $0 \%$ y $67 \%$ del tiempo) adecuadas a sus hijos, antes de iniciada la intervención. Consecuentemente, los tres niños presentaban, en sus casas, los mismos desajustes conductuales observados en el colegio: alta frecuencia de conducta agresiva verbal (entre el 10\% y $77 \%$ del tiempo), física (entre el $11 \%$ y $66 \%$ del tiempo) y oposicionista entre el $44 \%$ y $100 \%$ del tiempo). Durante la intervención las madres lograron disminuir gradualmente sus creencias irracionales hasta llegar a niveles mínimos en la última sesión (puntuaciones de 3 puntos o menos en todos los casos). Además las madres mejoraron el manejo conductual de sus hijos. Prueba de ello es que comenzaron a dar instrucciones y a aplicar contingencias de refuerzo y castigo de manera adecuada a sus hijos. Específicamente, aplicaron adecuadamente la alabanza verbal de conducta específica, la guía manual, el tiempo fuera de refuerzo y la restricción física pasiva. El desempeño apropiado de las madres en la entrega de instrucciones y aplicación de contingencias de refuerzo y castigo alcanzó el $100 \%$ del tiempo en la última sesión de intervención. Como era de esperarse los niños disminuyeron la frecuencia de la conducta agresiva verbal, física y oposicionista (estos tres tipos de conducta estuvieron ausentes durante la última sesión de intervención). Estos resultados se mantuvieron durante las mediciones de seguimiento realizadas a los 15 días y al mes después de finalizada la intervención.

\section{Análisis estadístico}

En el contexto escolar, se observa una disminución estadísticamente significativa en las creencias irracionales de las profesoras (tremendismo, autocondena y nosoportitis) entre las condiciones línea base y tratamiento. En el caso del tremendismo, se obtiene un valor $\mathrm{F}=316,162$ $(p=0,003)$, un tamaño del efecto observado de 0,994 , la potencia de prueba es de 1 y el contraste de varianzas de Levene sobre la igualdad de varianzas de error no es estadísticamente significativo $(p=0,427)$. A su vez, tanto para las condiciones de línea base y tratamiento existe un ajuste a la distribución normal con $\mathrm{Z}$ de Kolmogorov Smirnov de $0,354(\mathrm{p}=0,917)$ y de $0,471(\mathrm{p}=0,980)$ respectivamente. Habiéndose realizado contraste post Hoc DMS, se evidencia un valor estadísticamente significativo $(\mathrm{p}=0,003)$ a partir de la sesión 3 de tratamiento. En el caso de la autocondena de las profesoras se obtiene un valor $\mathrm{F}=19,857(\mathrm{p}=0,0001)$, tamaño del efecto de 0,908 y potencia de prueba de 0,182 . Por otro lado, el contraste de varianzas de Levene no es significativo $(p=0,577) y$, tanto para las condiciones de línea base y tratamiento existe ajuste a la distribución normal con $\mathrm{Z}$ de Kolmogorov Smirnov de 0,438 ( $\mathrm{p}=0,991)$ y de $0,380(p=0,997)$ respectivamente. El contraste post Hoc DMS indica un cambio significativo $(\mathrm{p}=0,020)$ a partir de la sesión 3 de tratamiento. En el caso de las creencias del tipo nosoportitis se obtiene un $\mathrm{F}=291,967(\mathrm{p}=0,035)$, un tamaño del efecto de 0,891 , una potencia de prueba de 0.572 , un contraste de varianzas de Levene no significativo $(\mathrm{p}=0,864)$ y un ajuste normal de las condiciones de línea base y tratamiento ( $\mathrm{Z}$ de Kolmogorov Smirnov de 0,401; $\mathrm{p}=0,996$ y 0,$438 ; \mathrm{p}=0,991$ respectivamente). Después de realizar contrastes post Hoc DMS, se evidencia una 


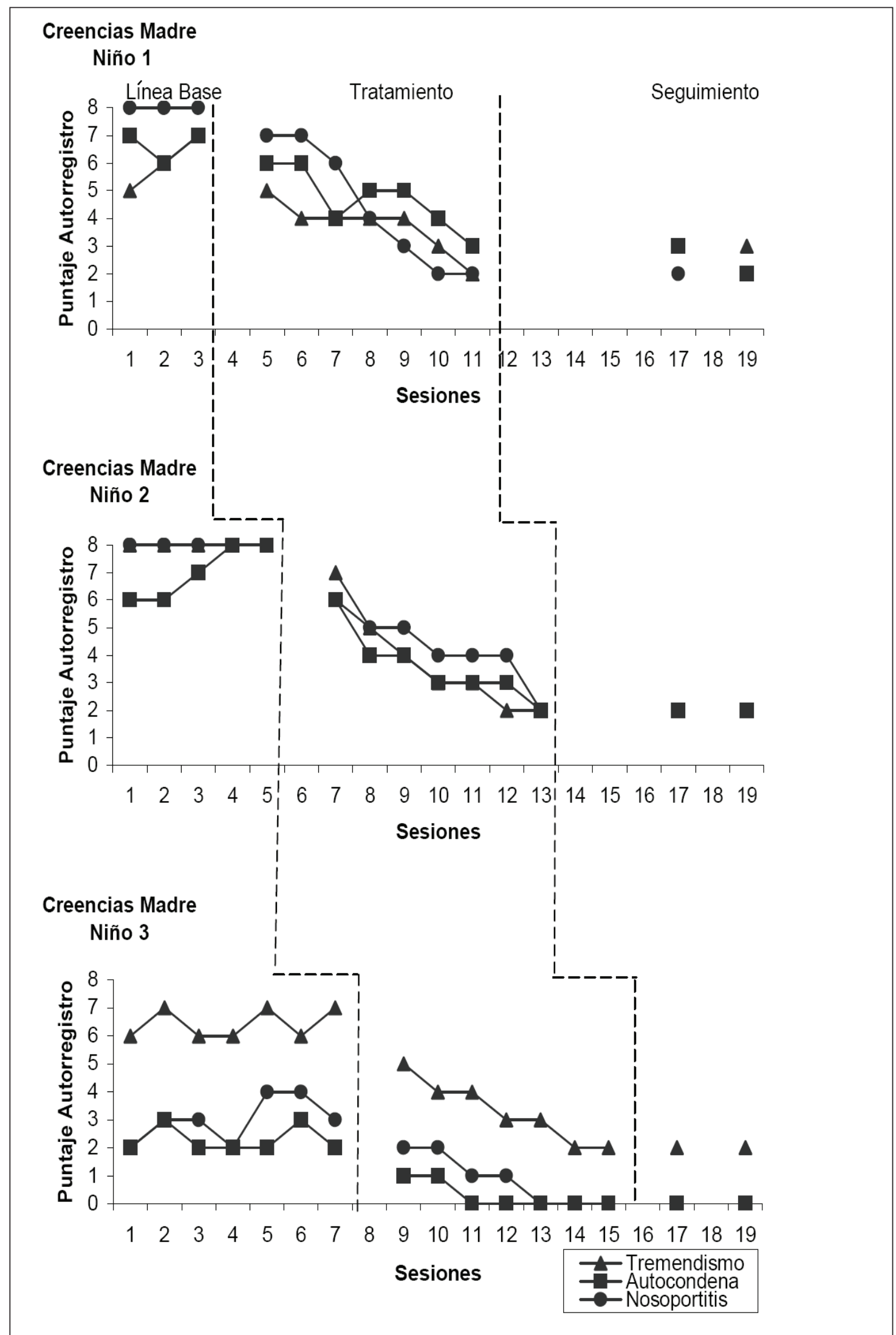

Figura 6: Cambios cognitivos en las madres en contexto familiar 


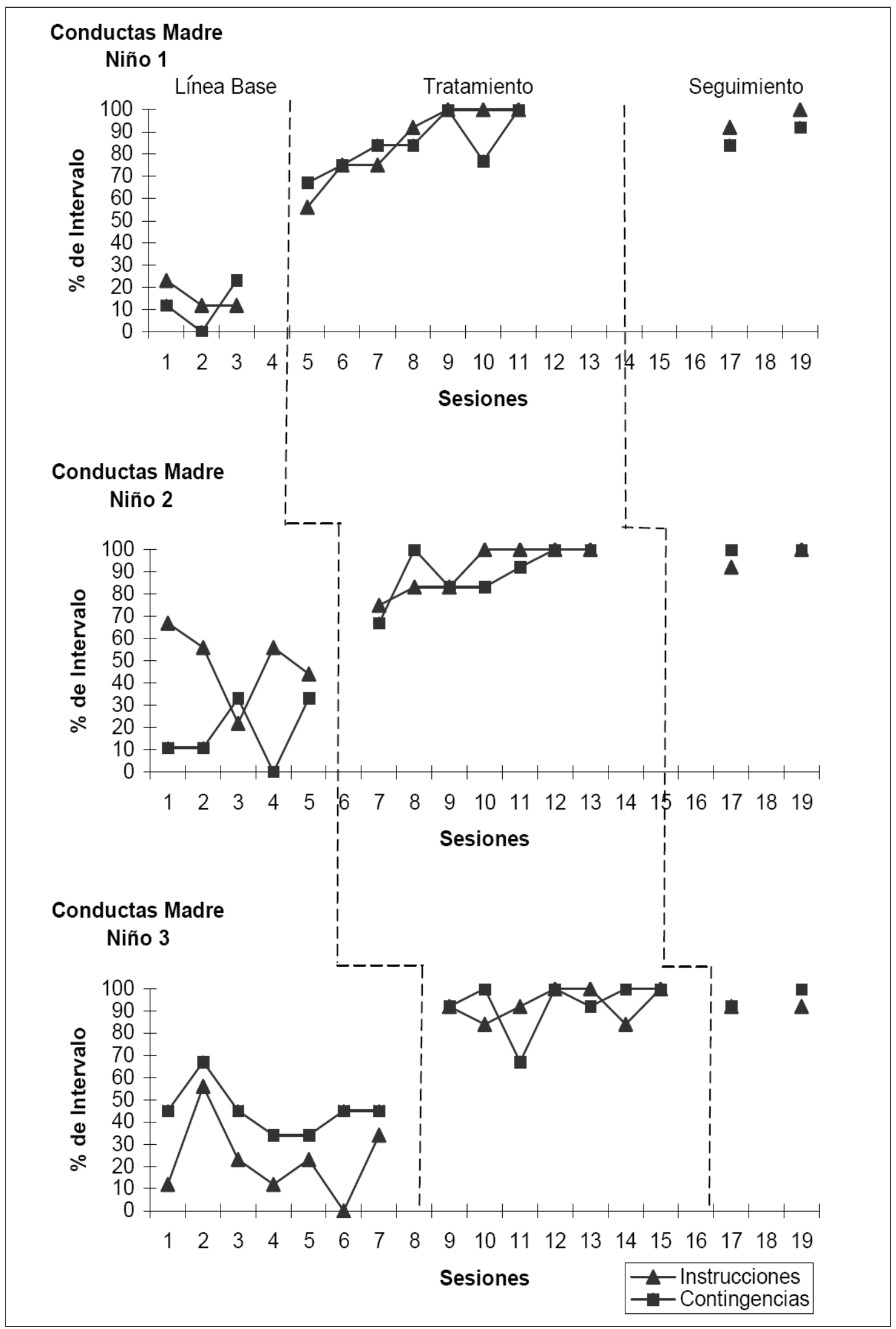

Figura 7: Cambios Conductuales en las madres en contexto familiar 


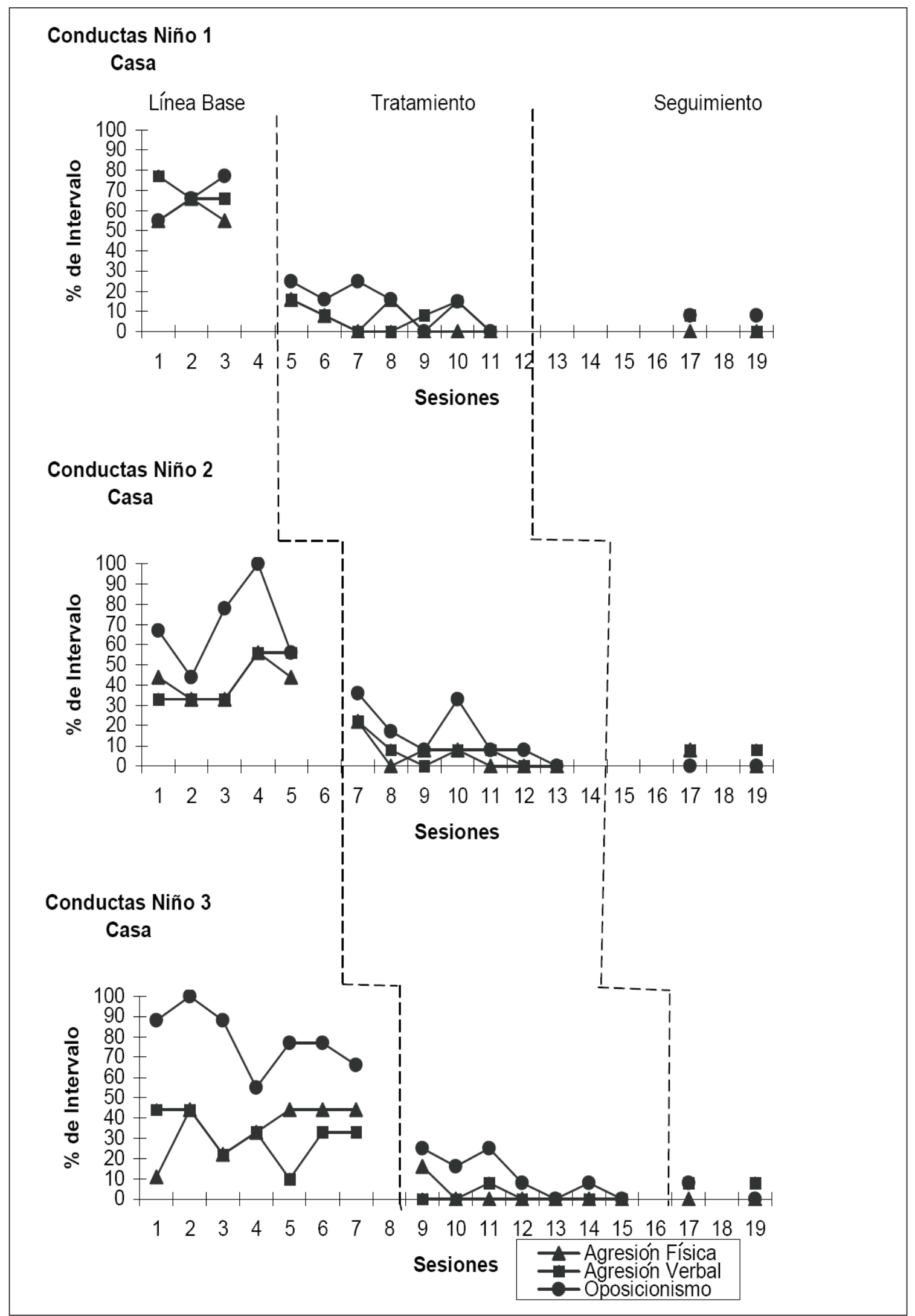

Figura 8: Cambios Conductuales en los niños en contexto familiar 
diferencia estadísticamente significativa $(\mathrm{p}=0,038)$ a partir de la sesión 2 de tratamiento.

En el caso de las instrucciones y contingencias aplicadas por las profesoras en el contexto escolar, se observa un aumento significativo entre las condiciones línea base $\mathrm{y}$ tratamiento. En el caso de las instrucciones el valor $\mathrm{F}=$ $234,941(p=0,000)$, tamaño del efecto observado de 0,892 , potencia de prueba de 0,998 y contraste de varianzas de Levene no significativo $(p=0,770)$. A su vez, tanto para las condiciones de línea base y tratamiento existe ajuste a la distribución normal con $\mathrm{Z}$ de Kolmogorov Smirnov de $0,501(p=0,963)$ y de $0,626(p=0,828)$ respectivamente. El contraste post Hoc DMS evidencia un valor significativo ( $p$ $=0,017)$ a partir de la sesión 3 de tratamiento. Sobre la correcta aplicación de contingencias se obtiene un valor $\mathrm{F}=26,798$ ( $p=0,000$ ), tamaño del efecto de 0,931 y potencia de prueba de 1. Por otro lado, el contraste de varianzas de Levene no es estadísticamente significativo $(p=0,643)$ y, tanto para las condiciones de línea base y tratamiento, existe ajuste a la distribución normal con $\mathrm{Z}$ de Kolmogorov Smirnov de $0,430(p=0,993)$ y de $0,380(p=0,999)$ respectivamente. Se evidencia un valor significativo $(p=0,030)$ a partir de la sesión 3 de tratamiento, según el contraste post Hoc DMS.

A nivel de los efectos del tratamiento en la conducta agresiva verbal, física y oposicionista de los niños en el contexto escolar, se observa una disminución significativa entre la línea base y el tratamiento. En el caso de la conducta agresiva verbal se obtiene un valor $F=20,939(p=0,045)$, un tamaño del efecto de 0,913 , potencia de prueba de 0,658 y el contraste de varianzas de Levene no es significativo $(p=0,345)$. A su vez, tanto para las condiciones de línea base y tratamiento existe ajuste a la distribución normal con Z de Kolmogorov Smirnov de 0,336 $(\mathrm{p}=0,996)$ y de $0,412(p=0,928)$ respectivamente. Habiéndose realizado contraste post Hoc DMS, se evidencia un valor estadísticamente significativo $(\mathrm{p}=0,026)$ a partir de la sesión 4 de tratamiento. En el caso de la agresión física de los niños en el contexto escolar se obtiene un valor $\mathrm{F}=13,710(\mathrm{p}=$ 0,000 ), tamaño del efecto de 0,873 y la potencia de prueba es de 1 . Por otro lado, el contraste de varianzas de Levene no es estadísticamente significativo $(p=0,381) y$, tanto para las condiciones de línea base y tratamiento existe ajuste a la distribución normal con $\mathrm{Z}$ de Kolmogorov Smirnov de $0,663(p=0,772)$ y de $0,638(p=0,810)$ respectivamente. El contraste post Hoc DMS evidencia un valor estadísticamente significativo $(p=0,036)$ a partir de la sesión 5 de tratamiento. En cuanto al oposicionismo de los niños en el contexto escolar se obtiene un valor $\mathrm{F}=16,554(\mathrm{p}=0,000)$. A su vez, el tamaño del efecto es de 0,999 , la potencia de prueba es de $1 \mathrm{y}$ el contraste de varianzas de Levene no es estadísticamente significativo $(p=0,423)$. A su vez, tanto para las condiciones de línea base y tratamiento existe ajuste a la distribución normal con $\mathrm{Z}$ de Kolmogorov Smirnov de $0,373(p=0,996)$ y de $0,369(p=0,992)$ respectivamente. Se observa cambio estadísticamente significativo $(p=0,018)$ a partir de la sesión 2 de tratamiento, según el contraste post Hoc DMS.

En el contexto familiar, se observa una disminución estadísticamente significativa en las creencias irracionales de las madres entre línea base y tratamiento. Para el tremendismo, se obtiene un valor $F=19,878(p=0,000)$, un tamaño del efecto de 0,909 , la potencia de prueba es de 1 y el contraste de varianzas de Levene no significativo $(p=0,629)$. A su vez, tanto para las condiciones de línea base y tratamiento existe un ajuste a la distribución normal con $\mathrm{Z}$ de Kolmogorov Smirnov de $0,533(p=0,939)$ y de $0,667(p=0,766)$ respectivamente. El contraste post Hoc DMS indica un valor estadísticamente significativo $(\mathrm{p}=0,015)$ a partir de la sesión 3 de tratamiento. Para la autocondena de las madres se obtiene un valor $\mathrm{F}=12,059(\mathrm{p}=0,000)$, tamaño del efecto de 0,851 , potencia de prueba de 1 , contraste de varianzas de Levene no significativo $(p=0,567)$ y un ajuste normal de las condiciones de línea base y tratamiento con $\mathrm{Z}$ de Kolmogorov Smirnov de $0,651(p=0,791)$ y de $0,667(p=0,766)$ respectivamente. El contraste post Hoc DMS indica un cambio significativo (p $=0,001)$ a partir de la sesión 4 de tratamiento. En el caso de nosoportitis se obtiene un $F=16,786(p=0,001)$, un tamaño del efecto de 0,894 , una potencia de prueba de 1 , un contraste de varianzas de Levene no significativo $(p=0,395)$ y un ajuste normal de las condiciones de línea base y tratamiento $(\mathrm{Z}$ de Kolmogorov Smirnov de 0,667, p = 0,766 en ambos casos). Después de realizar contrastes post Hoc DMS, se evidencia una diferencia estadísticamente significativa $(p=0,020)$ a partir de la sesión 3 de tratamiento.

En el caso de las instrucciones y contingencias de refuerzo y castigo aplicadas por las madres en el contexto familiar, se observa un aumento significativo entre las condiciones línea base y tratamiento. En el caso de las instrucciones el valor $\mathrm{F}$ obtenido es de 17,793 ( $\mathrm{p}=0,000)$, el tamaño del efecto observado de 0,899 , la potencia de prueba es de 1 y el contraste de varianzas de Levene no es significativo ( $p$ $=0,389$ ). A su vez, tanto para las condiciones de línea base $\mathrm{y}$ tratamiento existe ajuste a la distribución normal con $\mathrm{Z}$ de Kolmogorov Smirnov de 0,534 $(p=0,938)$ y de 0,541 $(p=0,932)$ respectivamente. El contraste post Hoc DMS evidencia un valor significativo $(p=0,044)$ a partir de la sesión 4 de tratamiento. Sobre la correcta aplicación de contingencias se obtiene un valor $\mathrm{F}=22,713(\mathrm{p}=0,000)$, tamaño del efecto de 0,919 y potencia de prueba de 0,896 . Por otro lado, el contraste de varianzas de Levene no es estadísticamente significativo $(p=0,147) y$, tanto para las condiciones de línea base y tratamiento existe ajuste a la distribución normal con $\mathrm{Z}$ de Kolmogorov Smirnov de $0,550(\mathrm{p}=0,922)$ y de $0,374(\mathrm{p}=0,999)$ respectivamente. Se evidencia un valor significativo $(\mathrm{p}=0,003)$ a partir de la sesión 1 de tratamiento, según el contraste post Hoc DMS.

A nivel de los efectos del tratamiento en la conducta agresiva verbal, física y oposicionista de los niños en el 
contexto familiar, se observa una disminución significativa entre la línea base y el tratamiento. En el caso de la conducta agresiva verbal se obtiene un valor $\mathrm{F}=9,628(\mathrm{p}=0,000)$, un tamaño del efecto de 0,828 , la potencia de prueba de 1 y el contraste de varianzas de Levene no es significativo $(\mathrm{p}=0,443)$. A su vez, tanto para las condiciones de línea base y tratamiento existe ajuste a la distribución normal con $\mathrm{Z}$ de Kolmogorov Smirnov de 0,511 $(\mathrm{p}=0,957)$ y de $0,653(p=0,787)$ respectivamente. Habiéndose realizado contraste post Hoc DMS, se evidencia un valor estadísticamente significativo $(p=0,033)$ a partir de la sesión 5 de tratamiento. En el caso de la agresión física de los niños en el contexto familiar se obtiene un valor $\mathrm{F}=19,280(\mathrm{p}=$ 0,001 ), tamaño del efecto de 0,906 y la potencia de prueba es de 0,767 . Por otro lado, el contraste de varianzas de Levene no es estadísticamente significativo $(p=0,824)$ $\mathrm{y}$, tanto para las condiciones de línea base y tratamiento existe ajuste a la distribución normal con $\mathrm{Z}$ de Kolmogorov Smirnov de $0,461(p=0,983)$ y de $0,621(p=0,836)$ respectivamente. El contraste post Hoc DMS evidencia un valor estadísticamente significativo $(p=0,000)$ a partir de la sesión 5 de tratamiento. En cuanto al oposicionismo de los niños en el contexto familiar se obtiene un valor $\mathrm{F}$ $=18,050(\mathrm{p}=0,033)$. A su vez, el tamaño del efecto es de 0,900 , la potencia de prueba es de 0,744 y el contraste de varianzas de Levene no es estadísticamente significativo (p $=0,392$ ). A su vez, tanto para las condiciones de línea base y tratamiento existe ajuste a la distribución normal con $Z$ de Kolmogorov Smirnov de 0, $517(\mathrm{p}=0,952)$ y de 0, 322 $(p=1,000)$ respectivamente. Se observa cambio estadísticamente significativo $(\mathrm{p}=0,016)$ a partir de la sesión $2 \mathrm{de}$ tratamiento, según el contraste post Hoc DMS.

\section{Análisis cualitativo de los cambios.}

En el contexto escolar las profesoras señalaron un aumento en su sensación de autoeficacia como educadoras y un aumento de su motivación por trabajar con los niños. Además señalaron que los niños que participaron del estudio experimentaron un notable cambio conductual que generó una mejora en el clima social de la sala de clases, y un aumento de su rendimiento.

En el contexto familiar las madres señalaron sentirse con más herramientas para tratar a sus hijos. Además reportaron sentir más emociones positivas en relación a sus hijos y una mejora de las relaciones al interior de la familia. Las madres reportaron realizar mayores actividades con los niños fuera del hogar (ej. ir con los niños a casas de amigos y familiares) que antes de la intervención no realizaban debido a la mala conducta de los niños. Finalmente reportan un cese de las prácticas de maltrato hacia los niños.

Por otro lado, se evidenció una mejora en la relación y una mayor coordinación entre las docentes y las madres.

Por su parte, los niños manifestaron mayor motivación a asistir al colegio y a participar de actividades familiares.
Por último, los niños aumentaron la frecuencia de comportamientos incompatibles con la agresividad para resolver sus conflictos.

\section{Discusión}

El programa de intervención fue exitoso ya que, tal como se esperaba, las madres y profesoras disminuyeron la frecuencia de las creencias irracionales asociadas a su relación con los niños. Además, mejoraron su desempeño en lo referido al manejo conductual de los niños, lo que coincidió con la disminución de la frecuencia de conductas agresivas verbales, físicas y oposicionistas en los preescolares. Los cambios favorables en las cogniciones de madres y profesoras, y en las conductas de niños, madres y profesoras han demostrado ser estadísticamente y clínicamente significativos.

Se considera que el éxito de esta intervención está dado por varios factores:

Primero, el haber trabajado en el hogar y en el colegio de los niños, lo que refuerza el planteamiento de Alberto \& Troutman (1999) referido a la necesidad de realizar un trabajo integral y en el contexto natural donde las conductas meta deben ser implementadas. Segundo, el haber incluido a madres y profesoras desde el primer momento para que sean ellas las que ejecuten los procedimientos de modificación conductual a los niños, ya que de este modo se interviene sobre la pauta de refuerzo mutuo entre los niños y los adultos a cargo de su formación. Esto confirma los planteamientos de Corsi, Barrera, Flores, Perivancich \& Guerra (2009), Rey (2006) y Trautmann (2008), sobre la utilidad del entrenamiento a padres y profesores. Tercero, el haber realizado el entrenamiento a las madres y profesoras incorporando tanto la dimensión cognitiva como la conductual ya que, como plantea el modelo de terapia cognitivo-conductual, los cambios en el comportamiento muchas veces deben estar asociados a un cambio en la forma de interpretar las situaciones (Ellis 1999, Ellis \& Lega 1993). Y en cuarto lugar, la planificación de la generalización de los resultados, donde en un comienzo se utilizó un programa de refuerzo continuo que gradualmente se fue transformando en un programa de refuerzo de razón variable, más resistente a la extinción.

Sobre la base de lo señalado se concluye que las intervenciones cognitivo-conductuales, como la descrita en esa investigación, se constituyen como una alternativa viable para la regulación de la agresividad entre preescolares. No obstante, es necesario destacar algunas limitaciones del estudio. En primer lugar, dado el número reducido de participantes, los resultados no pueden generalizarse al resto de la población de preescolares. Segundo, hay que señalar que el éxito de esta intervención se debe en gran medida al esfuerzo coordinado de diferentes agentes (directivos del establecimiento, profesoras, madres e investigadores), situación que en la gran mayoría de los casos es difícil de lograr. 


\section{Referencias}

Aguilar, C. \& Navarro, J. (2008). Análisis funcional e intervención con economía de fichas y contrato de contingencias en tres casos de conducta disruptiva en el entorno escolar. Revista Latinoamericana de psicología 40, 133- 139.

Alberto, P. \& Troutman, A. (1999). Applied behavior analysis for teachers. Londres: Prentice Hall.

Benítez, J.L. \& Justicia, F. (2006). El maltrato entre iguales: Descripción y análisis del fenómeno. Revista electrónica de Investigación Educativa 9, 151-170.

Caballo, V. (1998). Manual de técnicas de terapia y modificación de conducta. Madrid: Siglo XXI de España Editores.

Campaña, M.A., Fredes, V. \& Gutiérrez, L. (2008). Diseño de una escala para medir creencias irracionales asociadas al rol de madre y profesora. Manuscrito no publicado, Universidad del Mar, Chile.

Cooper, J. O., Heron, T. E. \& Heward, W. L. (2006). Applied behavior analysis. Second Edition. New York: Prentice Hall.

Corsi, E., Barrera, P., Flores, C., Perivancich, X. \& Guerra, C. (2009). Efectos de un programa combinado de técnicas de modificación conductual para la disminución de la conducta disruptiva y el aumento de la conducta prosocial en escolares chilenos. Acta Colombiana de Psicología 12, 67-76.

Corsi, E., Guerra, C. \& Plaza, H. (2007). Diseño, implementación y evaluación de un programa de manejo conductual para padres de niños con Síndrome de Asperger. Psicología Conductual,15, 253 - 266.

De la Barra, F., Toledo, V. \& Rodríguez, J. (2003). Estudio de salud mental en dos cohortes de niños escolares de Santiago Occidente: Predictores tempranos de problemas conductuales y cognitivos. Revista Chilena de Neuropsiquiatría, 41, 65-76.

Ellis, A. (1999). Una terapia breve más profunda y duradera. Barcelona: Paidós.

Ellis, A. \& Lega, L. (1993). Cómo aplicar algunas reglas básicas del método científico al cambio de las ideas irracionales sobre uno mismo, otras personas y la vida en general. Psicología Conductual, 1, $101-110$.

Forehand, R. \& Mc Mahon, R. (1981). A clinician's guide to parent training. New York: Guilford Press.

Foxx, R. (1982a). Increasing behaviors of severely retarded and autistic persons. Illinois: Research Press.

Foxx, R. (1982b). Decreasing behaviors of persons with severe retardation and autism. Illinois: Research Press.
Gutiérrez, H., Barrios, A., de Dios, M.J., Montero, I. \& del Barrio, C. (2008). The incident of peer bullying as multiple maltreatment among Spanish secondary school students. International Journal of Psychology and Psychological Therapy 8, 247-257.

Kellam, S., Rebok, G., Ialongo, N. \& Mayer, L. (1994). The course and malleability of aggressive behavior from early first grade into middle school: Results of a developmental epidemiologically based preventive trial. Journal of Child Psychology and Psychiatry, 35, 259- 281.

Livacic-Rojas, P., Espinoza, M. \& Ugalde, F. (2004). Intervenciones psicológicas basadas en la evidencia para la prevención de la delincuencia juvenil. Un programa de habilidades sociales en ambientes educativos. Terapia Psicológica, 22, 83-91.

Lozano, L. \& García, E. (2000). El rendimiento escolar y los trastornos emocionales y comportamentales. Psicothema 12, 340- 343.

Mestre, M.V., Tur, A.M., Samper, P., Nácher, J.M. \& Cortés, M.T. (2007). Estilos de crianza en la adolescencia y su relación con el comportamiento prosocial. Revista latinoamericana de psicología, 39, 211-225.

Nansel, T.R., Craig, W., Overpeck, M.D., Saluda, G. \& Ruan, J. (2004). Cross-national consistency in the relationship between bullying behaviors and psychosocial adjustment. Archives of Pediatrics and Adolescent Medicine 158, 730-736.

Ortega, R. \& Monks, C. (2005). Agresividad injustificada entre preescolares. Psicothema, 17, 453-458.

Pérez, V., Fernández, A. M., Rodríguez, J. \& De La Barra, F. (2005). Evaluación del efecto de una intervención conductual en la agresividad escolar. Terapia Psicológica, 23, 91-98.

Rey, C. (2006). Entrenamiento de Padres: Una revisión de sus principales componentes y aplicaciones. Revista Infancia, Adolescencia y Familia, $1,61-84$.

Schwartz, D., Dodge, K., Pettit, G. \& Bates, J. (1997). The early socialization of aggressive victims of bullying. Child Development 68, 665-675.

Trautmann, A. (2008). Maltrato entre pares o "Bullying": Una visión actual. Revista Chilena de Pediatría, 79, 13-20.

Trianes, M.V. \& Fernández-Figarés, C. (2001). Aprender a ser persona y a convivir: Un programa para secundaria. Bilbao: Descleé de Broker.

Urzúa, A., Ramos, M., Alday, C. \& Alquinta, A. (2010). Madurez neuropsicológica en preescolares: Propiedades psicométricas del test CUMANIN. Terapia Psicológica, 28, 13-25.

Zerón, A. M. (2002). Violencia Juvenil en el espacio escolar. Estudio sociológico comprensivo de la experiencia escolar. Tesis no publicada para obtener el grado de Doctora en Ciencias de la Educación, Pontificia Universidad Católica de Chile. 